\title{
Mininet-WiFi: Plataforma de Emulação para Redes sem Fio Definidas por Software
}

\author{
Ramon dos Reis Fontes ${ }^{1}$, Christian Esteve Rothenberg ${ }^{1}$ \\ ${ }^{1}$ Faculdade de Engenharia Elétrica e Computação (FEEC) \\ Universidade de Campinas (UNICAMP) \\ Campinas, São Paulo
}

\begin{abstract}
Software-Defined Wireless Networking (SDWN) has gained attraction as an appealing approach to decouple radio control functions, such as spectrum management, mobility management, and interference management, from the radio data plane devices. Despite diverse ongoing efforts to realize the vision of SDWN, many questions remain open from multiple perspectives, one of them being effective means to experiment and rapid prototyping of software components applicable to real-world deployments. To this end, realistic emulation of SDWN environments has the potential to boost research and development efforts by reusing existing protocol and application stacks while mimicking the behavior of real wireless networks. This thesis is centered around the feasibility of such an approach through the design, implementation, and evaluation of the Mininet-WiFi wireless network emulator.
\end{abstract}

Resumo. As redes sem fio definidas por software (SDWN) têm demonstrado ser uma abordagem atrativa e promissora que permite desacoplar as funções de controle de rádio do plano de dados, como gerenciamento de espectro, gerenciamento de mobilidade e gerenciamento de interferências. Apesar dos diversos esforços em prol de uma definição clara voltada para SDWN, muitas questões permanecem abertas a partir de múltiplas perspectivas, sendo uma delas a efetiva experiência e a prototipagem rápida de componentes de software candidatos e aplicáveis em implementações do mundo real. Para este fim, a emulação realista dos ambientes SDWN tem o potencial de impulsionar os esforços de pesquisa e desenvolvimento através da reutilização de protocolos e pilhas de aplicativos existentes, imitando o comportamento de redes sem fio reais. Nessa perspectiva, esta tese apresenta uma discussão sobre esse tema com foco no emulador de redes sem fio Mininet-WiFi, principal produto desta tese, com o objetivo de fornecer uma plataforma de experimentação de alta fidelidade para as redes sem fio.

\section{Introdução}

A necessidade de acompanhar o crescimento exponencial do tráfego móvel e as limitações relacionadas ao espectro estão tornando a infraestrutura sem fio cada vez mais caótica e densa. Como se não bastasse, as redes sem fio são muito sensíveis a mudanças, complexas de gerenciar e normalmente as soluções de controle não são flexíveis. Como consequência, esses problemas se tornam barreiras estruturais para várias tendências tecnológicas que demandem por maior conectividade e interoperabilidade. 
Sendo objeto de estudo em diversas pesquisas e propostas de soluções para os diferentes problemas e demandas inerentes às redes sem fio, as redes sem fio definidas por software (SDWN) [Jagadeesan et al. 2014, Costanzo et al. 2012] têm demonstrado ser uma solução promissora e capaz de mitigar as barreiras estruturais atuais e contribuir para um ecossistema inovador. Assim como nas redes definidas por software (SDN) [Kreutz et al. 2015], a proposta de SDWN é separar os planos de controle e dados, permitindo que as redes sem fio se tornem programáveis, abstraindo a infraestrutura subjacente de aplicativos e serviços de rede. Devido ao advento das SDWN, diversas pesquisas já foram realizadas e muitas estão em andamento. Isso significa que melhorias significativas na utilização de recursos estão por vir, junto com inovações e serviços personalizados aplicáveis no dia-a-dia. No entanto, além de ainda não haver uma arquitetura bem definida, não há plataforma experimental capaz de preencher todas as lacunas provenientes de estudos em SDWN. Plataformas essas que são historicamente importantes para a validação de propostas que tentam solucionar os mais diversos problemas.

\section{Motivação}

Apesar da existência de diversas pesquisas e soluções propostas para os diversos problemas e demandas acerca das redes sem fio, muitas questões ainda permanecem abertas a partir de múltiplas perspectivas. Assim, ferramentas de experimentação podem servir de suporte para um novo ponto de vista alternativo, permitindo a validação de conceitos e avaliação experimental dos benefícios. Para esse fim, a emulação das redes sem fio tem o potencial de impulsionar os esforços de pesquisa e desenvolvimento, através da reutilização das pilhas de protocolos e aplicativos existentes, imitando o comportamento de redes sem fio reais.

As redes sem fio são elementos essenciais em praticamente todos os lugares e há um ecossistema emergente de redes programáveis baseadas em desenvolvimentos de código aberto que vem transformando a forma como compreendemos o conceito de redes. Esse ecossistema emergente apresenta uma série de oportunidades sujeitas a alguns desafios, como, por exemplo, adaptação de protocolos como o OpenFlow (ou mesmo o desenvolvimento de novas soluções), para uso em redes de acesso celular e abstrações programáveis do plano de dados. Outros tópicos incluem temas como eficiência energética, gerenciamento de mobilidade, mitigação de interferência, técnicas de seleção de canal, etc.

No âmbito de plataformas experimentais, a emulação de redes [Fall 1999] tem sido uma técnica amplamente utilizada para avaliar desempenho, teste e depuração de protocolos. Mais acessível que testbeds e diferente das simulações, a emulação de redes permite executar código real de aplicações em condições realísticas de rede e computação. Em apoio à pesquisas sobre SDN, o emulador Mininet [Lantz et al. 2010] já permitia experimentos e ciclos de prototipagem rápida para as redes com fio. Ele é especialmente interessante para o ensino, pesquisa e reprodutibilidade para fins acadêmicos, bem como avaliação e pré-implantação da camada de controle para posterior implantação em ambientes reais, como ocorreu com BSN Labs ${ }^{1}$ e Google B4 [Jain et al. 2013]).

Sendo assim, considerando os potenciais e principalmente os casos de sucesso recentes para as redes com fio, a emulação de redes sem fio representa uma abordagem

\footnotetext{
${ }^{1}$ http://www.bigswitch.com/
} 
em potencial para alavancar pesquisas em redes sem fio, pois além de ser capaz de imitar o comportamento de redes sem fio reais, possibilita a utilização das pilhas de protocolos e aplicativos existentes, não exigindo esforço de aprendizagem adicional e muitas vezes desnecessário.

\section{Contribuições}

A principal contribuição desta tese é sem dúvidas o desenvolvimento do emulador Mininet-WiFi. Todas as demais contribuições estão diretamente relacionadas a este emulador, como, por exemplo, elaboração de diversos casos de uso demonstrando suas capacidades e áreas aplicáveis; e discussões sobre a nossa abordagem de pesquisa, correlacionando-a com experiências em torno da reproducibilidade de trabalhos científicos. Além disso, como forma de verificar a relevância deste trabalho junto a comunidade científica, elaboramos e disponibilizamos na página do código-fonte uma lista de citações e domínios de pesquisa onde o Mininet-WiFi tem sido aplicado. Em geral, as principais contribuições desta tese podem ser classificadas da seguinte forma:

- Fidelidade. Desenvolvimento do emulador sem fio Mininet-WiFi com suporte a diferentes modelos de propagação e que são facilmente customizados com o objetivo de fornecer resultados apropriados para diferentes cenários. Além disso, condições reais de rede também podem ser replicadas através da captura de traços de rede, com o objetivo de prover resultados mais próximos aos obtidos em ambientes reais;

- Casos de uso. Diversos tópicos de pesquisa foram explorados em nossas publicações, tais como: (i) wireless bicasting; (ii) integração entre ambientes físico e virtual; (iii) reprodução de condições de rede; (iv) multipath TCP; (v) encaminhamento baseado no SSID e protocolo OpenFlow; (vi) redes veiculares; (vii) segurança; (viii) network slicing; entre outros. Os casos de uso não ficam limitados aos nossos trabalhos. Tópicos de pesquisa identificados por outros autores incluem fog computing; internet das coisas; gestão de energia; etc;

- Reproducibilidade. Neste aspecto, duas contribuições principais podem ser destacadas: a) a de permitir ao emulador prover realismo suficiente para reproduzir pesquisas de terceiros; b) e a de tornar nossas pesquisas reprodutíveis. Como consequência da prática da reprodutibilidade com o Mininet-WiFi, aprendemos e aprimoramos nossos conceitos e sempre trabalhamos em prol de uma pesquisa reprodutível, onde outros usuários pudessem reproduzir os resultados obtidos em nossas pesquisas ${ }^{2}$. Nesse sentido, uma série de experiências foram identificadas e adquiridas, tais como: (i) o código aberto melhora as chances de reprodutibilidade direta e indireta, e, consequentemente, (ii) traz maior visibilidade, uma vez que as chances de que outros pesquisadores utilizem as soluções propostas são maiores; (iii) além de trazer maior credibilidade aos resultados alcançados, principalmente quando submetido a avaliação para revisores; (iv) também traz melhoria nos hábitos, onde uma atenção especial é dada à qualidade do código; e finalmente nos deixa mais atentos acerca do nosso (v) fluxo de trabalho, uma vez que estamos mais cuidadosamente preocupados em fornecer resultados confiáveis;

- Educacional. A possibilidade de utilizar uma abordagem real e por ser relativamente simples de se trabalhar, traz uma outra vantagem para o Mininet-WiFi:

\footnotetext{
${ }^{2}$ https://github.com/ramonfontes/reproducible-research/
} 
permite que seja utilizado em cursos de redes de computadores, onde pode ser aplicado para diversos propósitos, desde os conceitos básicos de redes até os mais avançados. Dito isto, listamos abaixo alguns dos cursos onde Mininet-WiFi tem sido utilizado.

1. Summer School. Software-Defined Wireless Networking (SDWN): From Theory to Practice with Mininet-WiFi. International Summer School on Latency Control for Internet of Services. 26 June - 30, June 2017, Karlstad, Sweden.

2. Curso de extensão (INF556). Software-Defined Networking. Unicamp, 2016, 2017 e 2018.

3. Curso de graduação (IA376). Advanced Topics in Networking: Laboratório de Redes Softwarizadas/Network Softwarization Lab. Unicamp, 2017, 2018.

4. Curso de graduação (EA080). Laboratory of Network Computing. Unicamp, 2018.

5. Curso técnico. IFBA, 2017/2018.

6. Hackaton. Universidade de Memphis, 2017. Como resultado deste hackaton, uma solução para Named Data Networking (NDN) foi desenvolvido ${ }^{3}$ a partir do Mininet-WiFi.

7. Diversos programas de pós-graduação também têm referenciado o Mininet-WiFi em suas chamadas de vagas para mestrado e doutorado.

\subsection{Outras contribuições}

Outras informações relevantes sobre o desenvolvimento do Mininet-WiFi incluem: (i) como consequência de trabalhos em torno do SUMO (Simulation of Urban MObility) [Sumo 2017], o Mininet-WiFi foi extendido para suportar classes deste simulador, viabilizando pesquisas em redes veiculares; (ii) extensão similar foi desenvolvida com o Sflow ${ }^{4}$, tecnologia que permite monitorar dispositivos de rede; (iii) até onde sabemos, fomos os primeiros a criar aplicações para controladores SDN capazes de filtrar e processar beacons. Controladores como $\mathrm{Pox}^{5}$ e Ryu ${ }^{6}$ foram extendidos; (iv) melhorias para o Wmediumd ${ }^{7}$ também foram propostas e implementadas, incluindo a implementação de novos modelos de propagação.

\section{Trabalhos relacionados}

Um pesquisador tem tipicamente várias possibilidades para avaliar e validar pesquisas, protocolos de rede, bem como realizar análises, entre outros. Simuladores, emuladores e testbeds são os principais métodos de avaliação que auxiliam os pesquisadores em suas tarefas. Em relação à aplicação real, todos esses métodos de avaliação são muito diferentes em seu grau de abstração. Portanto, realizamos o levantamento de trabalhos relacionados para identificar suas vantagens e desvantagens, afim de demonstrar o porque o Mininet-WiFi pode ser o preferido se comparado aos demais trabalhos.

Primeiro, identificamos as diferenças entres os três principais métodos de experimentação e apresentamos as vantagens dos emuladores em relação aos demais. Então, dentre os emuladores identificados, expomos as justificativas que fazem o MininetWiFi ter vantagem se comparado aos outros emuladores. Em geral, o emulador que mais se aproximou do Mininet-WiFi, o ns3/DCE [Mancini et al. 2014], exige alterações em

\footnotetext{
${ }^{3}$ https://netwisdom.cs.memphis.edu/gitlab/nmgordon/mini-ndn-wifi

${ }^{4}$ https://sflow.org

${ }^{5}$ https:/github.com/ramonfontes/pox

${ }^{6}$ https://github.com/ramonfontes/ryu

${ }^{7}$ https://github.com/ramonfontes/wmediumd
} 
código-fonte de aplicações de terceiros, o que acaba resultando em outros problemas mais sérios. Por exemplo, pode-se levar anos para que o ns3/DCE permita experimentação de determinadas aplicações e/ou tecnologias; e, normalmente, os códigos e procedimentos utilizados não podem ser replicados em ambientes reais. É importante destacar também que alguns problemas e limitações também foram identificados no Mininet-WiFi. Para os problemas e limitações, apontamos cenários ou situações onde o Mininet-WiFi poderia não corresponder como esperado, a exemplo de cenários onde há grande quantidade de nós ou alta demanda de processamento.

\section{Mininet-WiFi}

Desenvolvido na linguagem de programação Python, o Mininet-WiFi é uma plataforma de emulação para redes sem fio e redes sem fio definidas por software. O suporte ao WiFi vem do módulo nativo do kernel do sistema operacional Linux, o mac80211 hwsim. Deste a sua criação, que ocorreu em 2008, este módulo vem sendo constantemente atualizado pela comunidade de desenvolvedores do kernel do sistema operacional Linux. Ele suporta uma grande variedade de protocolos 802.11, como IEEE 802.11a,b,g,n,ac,ax, etc. Como consequência, o Mininet-WiFi também os suporta. As atualizações neste módulo vem acompanhando as evoluções do mac80211, ao qual o módulo mac80211 hwsim é baseado, e que hoje talvez seja a principal framework utilizada por desenvolvedores de drivers sem fio para sistemas baseados em Linux.

Como tem sido desenvolvido em cima do conceito de Container-Based Emulation (CBE), uma das principais categorias de emulação de rede que usa containers leves, cada nó virtual emulado é simplesmente um grupo de processos do chamado user space do sistema operacional. Além disso, uma vez que compartilha o mesmo kernel do sistema operacional onde está sendo executado, basicamente qualquer aplicação compatível com sistemas operacionais Linux pode ser utilizado com o Mininet-WiFi. Isso significa que ao utilizar o Mininet-WiFi, o usuário não precisa se preocupar em adaptar ou codificar aplicações, uma vez que não há necessidade de alterações na forma de funcionamento delas.

Embora a proposta inicial para o desenvolvimento do Mininet-WiFi tenha sido Wi-Fi, ele também vem sendo utilizado em pesquisas que envolvam outras tecnologias de redes sem fio. Além de permitir simulações para redes LTE (Long Term Evolution), extensões para outras tecnologias já foram iniciadas, como o 6LoWPAN, que vem sendo implementado nativamente para sistemas Linux através do módulo mac802154_hwsim. O objetivo agora é fazer com que o Mininet-WiFi suporte mais tecnologias sem fio para que tendências tecnológicas como IoT possam ser experimentadas através deste emulador.

\subsection{Fidelidade na Emulação}

O Mininet-WiFi foi pensado desde início com o objetivo de ser um emulador que provesse alta fidelidade. Isso significa que o meio sem fio emulado deveria ser representado da forma mais fiel possivel se comparado ao ambiente real. Inicialmente, a única forma de emular o meio sem fio era através da ferramenta TC (Traffic Control), um pequeno programa que permite manipular atributos de rede das interfaces, como largura de banda suportada e latência. Após algum tempo de desenvolvimento, o Wmediumd ${ }^{8}$, um simulador do meio sem fio para o módulo mac80211 hwsim foi extendido para suportar o

\footnotetext{
${ }^{8}$ https://github.com/patgrosse/wmediumd
} 
Mininet-WiFi. Parte do código-fonte do módulo mac80211 hwsim, inclusive, tem sido desenvolvido para atuar em conjunto com o Wmediumd.

Para verificarmos o quão próximo da realidade o Mininet-WiFi poderia chegar, realizamos pesquisas no INRIA (Institut National de Recherche en Informatique et en Automatique) juntamente com pesquisadores locais. Duas pesquisas específicas foram realizadas: reprodução de condições de rede e realismo na emulação do canal sem fio. Para tanto, utilizamos o R2 $\mathrm{Lab}^{9}$, um testbed sem fio que nos permitiu comparar os resultados obtidos no Mininet-WiFi com aqueles obtidos através do testbed. O R2lab consiste de um conjunto de 37 nós organizados em uma câmara que mede aproximadamente $90 \mathrm{~m}^{2}$. Por ser uma câmara anecóica, o R2lab provê ambiente adequado para experimentações com alto grau de fidelidade.

\subsubsection{Reprodução de condições de rede}

A reprodução de condições de rede consiste na capacidade do Mininet-WiFi de alterar dinamicamente os parâmetros dos links de rede, como largura de banda, perda de pacotes e latência. O meio sem fio é conhecido pelas frequentes variações nas condições de rede devido a vários motivos, como mobilidade e interferências causadas por outras fontes, que acabam contribuindo para flutuações no tráfego de dados. Ser capaz de reproduzir condições reais de rede com base em observações de tráfego de ambientes reais, dentre outras coisas, é útil para poder prever o desempenho da rede sob certas condições, explicar o comportamento de rede observado e realizar comparações justas entre implementações de algoritmos alternativos sujeitos à representação da rede física.

Em pesquisa realizada no R2Lab, capturamos traços de rede a partir da transferência de dados realizada neste testbed e replicamos esses traços no Mininet-WiFi para que comparações de desempenho entre os dois ambientes, físico e virtual, pudessem ser realizadas. Por exemplo, durante a tranferência de dados realizada entre nós físicos do R2Lab, capturamos traços de largura de banda e latência. Após isso, com os traços salvos, pudemos replicá-los no Mininet-WiFi e obter resultados muito próximos aos obtidos no R2Lab.

\subsubsection{Realismo na emulação do canal sem fio}

O objetivo de identificar o realismo da emulação do canal sem fio foi verificar se os modelos de propagação suportados pelo Mininet-WiFi também poderiam ser validados ou confrontados com o comportamento observado no R2Lab. Por exemplo, a partir de um nó de referência, medimos o nível de sinal recebido por outro nó em diferentes distâncias. Como o R2Lab é um testbed anecóico e consequentemente livre de interferências externas, seria normal esperar que modelos de propagação como o free space pudesse corresponder à altura ao comportamento observado no R2Lab. Porém, esse não foi o caso. Cada ambiente possui características específicas e provavelmente únicas que nem sempre é fielmente representado pelo modelo de propagação teoricamente mais apropriado. E pudemos, através do Mininet-WiFi, identificar o modelo de propagação e customizá-lo de forma a representar com maior fidelidade possível o ambiente do R2Lab,

\footnotetext{
${ }^{9}$ https://r2lab.inria.fr
} 


\section{Considerações finais}

Os resultados desta tese foram publicados em algumas conceituadas revistas e conferências na área de Redes de Computadores, a exemplo do SIGCOMM. Mais recentemente, um livro de autoria própria que reúne conceitos e estudos de casos explorados em nossos trabalhos foi publicado sob o título "Emulando Redes sem Fio com Mininet-WiFi" - ISBN 978-65-900571-0-5 ${ }^{10}$. Na tabela 1 é possível identificar os diferentes recursos explorados nos estudos de casos e os ponteiros para as publicações onde eles foram utilizados. Através desta tabela é possível identificar também o crescente número de recursos utilizados nas publicações mais recentes, uma vez que estudos de casos de maior complexidade normalmente exigem a combinação de vários recursos.

Tabela 1. Recursos explorados em casos de uso

\begin{tabular}{|c|c|c|c|c|c|c|c|c|c|c|c|}
\hline & 1 & 2 & 3 & 4 & 5 & 6 & 7 & 8 & 9 & 10 & 11 \\
\hline Bicasting & & & & & $\sqrt{ }$ & & & & & & $\checkmark$ \\
\hline MPTCP & & & & & $\sqrt{ }$ & & & & & $\checkmark$ & \\
\hline Authentication & $\checkmark$ & $\checkmark$ & $\checkmark$ & & & & & & & & \\
\hline Bgscan & $\checkmark$ & $\checkmark$ & & & & & & & & & \\
\hline IEEE 802.11r & $\sqrt{ }$ & $\checkmark$ & $\checkmark$ & & & & & & & & \\
\hline IEEE 802.1x & $\checkmark$ & & $\checkmark$ & & & & & & & & \\
\hline Hybrid Physical/Virtual Network & & & & & $\sqrt{ }$ & & & $\checkmark$ & $\checkmark$ & & \\
\hline VANETs & $\checkmark$ & $\checkmark$ & & & & $\checkmark$ & & & & & \\
\hline MEC & & $\checkmark$ & & & & & & & & & \\
\hline Network Slicing & $\checkmark$ & & & & & & & & & & \\
\hline OpenFlow Protocol & $\checkmark$ & $\sqrt{ }$ & $\checkmark$ & & $\sqrt{ }$ & $\sqrt{ }$ & $\sqrt{ }$ & $\checkmark$ & $\checkmark$ & $\checkmark$ & $\checkmark$ \\
\hline
\end{tabular}

1. Campolo, C., Fontes, R., Molinaro, A., Rothenberg, C., Iera, A. Slicing on the Road: Enabling the Automotive Vertical through $5 G$ Network Softwarization. Sensors. doi 10.3390/s18124435, 2018. Qualis A1.

2. Al-Badarneh, J. Jararweh, Y. Al-Ayyoub, M. Fontes, R., Al-Smadi, M., Rothenberg, C. Cooperative Mobile Edge Computing System for VANET-Based Software Defined Content Delivery. Elsevier Computers and Electrical Engineering, 2018. Qualis B1.

3. Fontes, R., Rothenberg, C. On the Krack Attack: Reproducing Vulnerability and a SoftwareDefined Mitigation Approach. WCNC. Barcelona, Spain, 2018. Qualis B1.

4. Campolo, C., Fontes, R., Iera, A., Rothenberg, C., Molinaro, A. Towards $5 G$ Network Slicing for the V2X Ecosystem. Workshop on Advances in Slicing for Softwarized Infrastructures. NETSOFT. Montreal, Canada, 2018.

5. Fontes, R., Mahfoudi, M., Dabbous, W., Turletti T., Rothenberg, C. How Far Can We Go? Towards Realistic Software-Defined Wireless Networking Experiments. doi: 10.1093/comjnl/bxx023. Oxford University Press. The Computer Journal, 2017. Qualis A2.

6. Fontes, R., Campolo, C., Rothenberg, C., Molinaro, A. From Theory to Experimental Evaluation: Resource Management in Software-Defined Vehicular Networks. doi: 10.1109/access.2017.2671030. Journal: IEEE Access, 2017. Qualis B2.

7. Badarneh, J. Jararweh, Y., Al-Ayyoub, M., Al-Smadi M., Fontes, R. Software Defined Storage for Cooperative Mobile Edge Computing System. SDS. Valencia, Spain, 2017.

8. Fontes, R., Rothenberg, C. Mininet-WiFi: A Platform for Hybrid Physical-Virtual SoftwareDefined Wireless Networking Research.. SIGCOMM. doi: 10.1145/2934872.2959070. ACM, Florianopolis, Brazil. 2016. Qualis A1.

9. Fontes, R., Rothenberg, C. Mininet-WiFi: Emulação de Redes Sem Fio Definidas por Software com suporte a Mobilidade. SBRC. Salvador, BA, Brazil. June, 2016. Qualis B2.

10. Fontes, R., Afzal, S., Brito, S., Santos, M., Rothenberg, C. Mininet-WiFi: Emulating Softwaredefined Wireless Networks. CNSM. doi: 10.1109/CNSM.2015.7367387, IEEE Computer Society. Washington, DC, USA. 2015. Best Paper Award. (+100 citações).

${ }^{10}$ https://github.com/ramonfontes/mn-wifi-book-pt 
11. Fontes, R., Rothenberg, C. Towards an Emulator for Software-Defined Wireless Networks. SwitchOn, Sao Paulo, SP, Brazil. October, 2015.

Impacto. O Mininet-WiFi tem sido utilizado por diversos pesquisadores em diferentes tópicos de pesquisa, tais como: controle no consumo de energia, segurança, qualidade do serviço, cloud computing, software-defined storage, redes veiculares, internet das coisas, etc. Outros tópicos de pesquisa incluem o desenvolvimento de controladores SDN com suporte a redes sem fio e autenticação. Como destacado na lista de publicações acima, uma das publicações já conta com mais de 100 citações, sendo que de acordo com nosso levantamento, ${ }^{11}$ mais de $80 \%$ das citações, de fato, utilizaram o Mininet-WiFi no suporte às suas pesquisas. Os demais $20 \%$ incluem citações no sentido de utilização futura do Mininet-WiFi para validação das pesquisas e também como sendo um trabalho relacionado por ser uma proposta de pesquisa similar. Entre as citações incluem capítulo de livro intitulado de "Python Network Programming Cookbook" (Second Edition, ISBN: 9781786463999); além de diversos artigos, teses, dissertações e relatórios técnicos. Ao todo, as nossas publicações já contam com mais mais de 150 citações. Um catálogo aberto dos trabalhos que utilizaram o Mininet-WiFi, com detalhes sobre os autores, domínios de pesquisa, código-fonte e endereço, está publicamente disponível para consulta através do repositório do código-fonte do Mininet-WiFi.

Além das citações, outro resultado bastante relevante é o tamanho e atividade da comunidade de usuários (mininet-wifi-discuss@googlegroups.com), que hoje conta com aproximadamente 600 membros e cerca de 700 tópicos, criados para dúvidas e sugestões acerca do desenvolvimento e funcionamento do Mininet-WiFi. Embora projetos, como projetos de doutorado, façam parte de um ciclo, onde a fase de conclusão seja comum para a maioria dos casos, estamos certos de que o ciclo do projeto relacionado ao MininetWiFi está longe de acabar. Além da grande quantidade de clones que são realizados do código do Mininet-WiFi toda semana, cada vez mais surgem novas citações com o uso efetivo deste emulador. Porém, a nossa constatação vem principalmente da comunidade científica. Demandas acerca de novas implementações estão sempre surgindo, a exemplo de tendências tecnológicas como a Internet das Coisas. Citações nesse sentido já começaram a aparecer, além de implementações iniciais, como o suporte ao 6LowPAN.

\section{Referências}

Costanzo, S. et al. (2012). Software Defined Wireless Networks: Unbridling SDNs. In EWSDN, pages 1-6. IEEE.

Fall, K. (1999). Network emulation in the vint/ns simulator. In Proceedings of the fourth IEEE Symposium on Computers and Communications, pages 244-250.

Jagadeesan, N. A., Krishnamachari, B., et al. (2014). Software-defined networking paradigms in wireless networks: A survey. ACM Comput. Surv., 47(2):27:1-27:11.

Jain, S. et al. (2013). B4: Experience with a globally-deployed software defined wan. SIGCOMM Comput. Commun. Rev., 43(4):3-14.

Kreutz, D. et al. (2015). Software-defined networking: A comprehensive survey. Proceedings of the IEEE.

Lantz, B. et al. (2010). A network in a laptop: Rapid prototyping for software-defined networks. In Proceedings of the 9th ACM SIGCOMM Workshop on HotNets.

Mancini, E. P. et al. (2014). Demo abstract: Realistic evaluation of kernel protocols and software defined wireless networks with dce/ns-3. In MSWiM, pages 335-337, New York, USA. ACM.

Sumo (2017). Simulation of urban mobility (SUMO). http://www.sumo.dlr.de. Accessed 07 January 2019.

\footnotetext{
${ }^{11}$ https://goo.gl/K7YvZR
} 